\title{
THE STABILITY OF THE COSINE EQUATION ${ }^{1}$
}

\author{
JOHN A. BAKER
}

\begin{abstract}
If $\delta>0, G$ is an abelian group and $f$ is a complex-valued function defined on $G$ such that $|f(x+y)+f(x-y)-2 f(x) f(y)|<\delta$ for all $x, y \in G$, then either $|f(x)|<(1+\sqrt{1+2 \delta}) / 2$ for all $x \in G$ or $f(x+y)+f(x-y)=$ $2 f(x) f(y)$ for all $x, y \in G$.
\end{abstract}

1. Introduction. Questions concerning the stability of functional equations seem to have been first raised by S. M. Ulam (see [4, Chapter VI], and [2]). D. H. Hyers [2] showed that if $\delta>0$ and $f: E \rightarrow E^{\prime}$, with $E$ and $E^{\prime}$ Banach spaces, such that $\|f(x+y)-f(x)-f(y)\|<\delta$ for all $x, y \in E$ then there exists a unique $g: E \rightarrow$ $E^{\prime}$ such that $g(x+y)=g(x)+g(y)$ and $\|f(x)-g(x)\|<\delta$ for all $x, y \in E$. In response to a problem of E. Lukacs in statistics, J. Lawrence, F. Zorzitto and the author proved in [1] that if $\delta>0, V$ is a rational vector space and $f$ is a real-valued function defined on $V$ such that $|f(x+y)-f(x) f(y)|<\delta$ for all $x, y \in V$ then either $|f(x)| \leqslant(1+\sqrt{1+4 \delta}) / 2$ for all $x \in V$ or $f(x+y)=f(x) f(y)$ for all $x, y \in V$. The main purpose of this paper is to prove an analogous result for the "cosine" equation

$$
f(x+y)+f(x-y)=2 f(x) f(y) .
$$

We will also extend and greatly simplify the results of [1].

2. Approximate exponentials. We will need the following theorem which generalizes the results of [1].

THEOREM 1. Let $\delta>0$, let $S$ be a semigroup and let $f$ be a complex-valued function defined on $S$ such that $|f(x y)-f(x) f(y)|<\delta$ for all $x, y \in S$. Then either $|f(x)|<$ $(1+\sqrt{1+4 \delta}) / 2=: \varepsilon$ for all $x \in S$ or $f(x y)=f(x) f(y)$ for all $x, y \in S$.

Proof. Notice that $\varepsilon^{2}-\varepsilon=\delta$ and $\varepsilon>1$. Suppose there exists $a \in S$ such that $|f(a)|>\varepsilon$, say $|f(a)|=\varepsilon+p$ for some $p>0$. Then

$$
\begin{aligned}
\left|f\left(a^{2}\right)\right| & \geqslant\left|f(a)^{2}-\left(f(a)^{2}-f\left(a^{2}\right)\right)\right| \geqslant\left|f(a)^{2}\right|-\left|f(a)^{2}-f\left(a^{2}\right)\right| \\
& \geqslant|f(a)|^{2}-\delta=(\varepsilon+p)^{2}-\delta=(\varepsilon+p)+(2 \varepsilon-1) p+p^{2}>\varepsilon+2 p .
\end{aligned}
$$

By induction, $\left|f\left(a^{2^{n}}\right)\right|>\varepsilon+(n+1) p$ for all $n=1,2, \ldots$

For every $x, y, z \in S$,

$$
|f(x y z)-f(x y) f(z)| \leqslant \delta \text { and }|f(x y z)-f(x) f(y z)|<\delta
$$

Received by the editors July 13, 1979.

AMS (MOS) subject classifications (1970). Primary 39B20; Secondary 39B50, 26 D05.

Key words and phrases. Functional equation, cosine equation, stability.

${ }^{1}$ Research supported by NSERC Grant A7153. 
so $|f(x y) f(z)-f(x) f(y z)|<2 \delta$. Hence

$$
\begin{aligned}
\mid f(x y) f(z)- & f(x) f(y) f(z) \mid \\
& <|f(x y) f(z)-f(x) f(y z)|+|f(x) f(y z)-f(x) f(y) f(z)| \\
& <2 \delta+|f(x)| \delta
\end{aligned}
$$

or

In particular

$$
|f(x y)-f(x) f(y)| \cdot|f(z)|<2 \delta+|f(x)| \delta .
$$

$$
|f(x y)-f(x) f(y)|<(2 \delta+|f(x)| \delta) /\left|f\left(a^{2^{n}}\right)\right|
$$

for all $x, y \in S$ and all $n=1,2, \ldots$ Letting $n \rightarrow+\infty$ shows that $f(x y)=$ $f(x) f(y)$ for all $x, y \in S$.

The crucial step in this proof is the first one where we use the fact that $|z w|=|z||w|$ for all complex $z$ and $w$. Because the quaternions and Cayley numbers enjoy the same property, it is easy to see that Theorem 1 is also true for functions $f$ with values in the quaternions or Cayley numbers. For real normed algebras for which the norm is not multiplicative, the situation is not so simple as the following example shows. Let $\delta>0$, choose $\varepsilon>0$ so that $\left|\varepsilon-\varepsilon^{2}\right|=\delta$ and let

$$
f(x)=\left[\begin{array}{cc}
e^{x} & 0 \\
0 & \varepsilon
\end{array}\right] \text { for } x \text { real. }
$$

Then, with the usual matrix norm, $\|f(x+y)-f(x) f(y)\|=\delta$ for all real $x$ and $y, f$ is unbounded but it is not true that $f(x+y)=f(x) f(y)$ for all real $x$ and $y$.

3. The cosine equation. The following theorem was proved by Kannappan [3]. The proof given here uses ideas of Wilson [5] and motivates the proof of our stability result.

THEOREM 2. Let $G$ be an additive abelian group and let $f$ be a complex-valued function defined on $G$. Then $f$ satisfies (1) for all $x, y \in G$ if and only if there exists $a$ complex-valued function $m$ defined on $G$ such that

$$
f(x)=\{m(x)+m(-x)\} / 2 \text { for } x \in G
$$

and

$$
m(x+y)=m(x) m(y) \text { for all } x, y \in G .
$$

Proof. It is easy to check that (2) and (3) imply (1), so assume $f$ satisfies (1) for all $x, y \in G$.

Put $y=0$ in (1) to conclude that either $f \equiv 0$ or $f(0)=1$. If $f \equiv 0$ then (2) and (3) hold with $f=m$. Thus we may suppose $f(0)=1$. Now replace $y$ by $-y$ in (1) and compare the resulting equation with (1) to conclude that $f(-y)=f(y)$, i.e. $f$ is even.

If $f(G) \subseteq\{1,-1\}$ then $f(x+y)=f(x-y)=1$ if $f(x) f(y)=1$ and $f(x+y)=$ $f(x-y)=-1$ if $f(x) f(y)=-1$. In this case $f(x+y)=f(x) f(y)$ for all $x, y \in G$ so that (2) and (3) hold with $m=f$.

To complete the proof we may assume $f(a)^{2} \neq 1$ for some $a \in G$. However 
$f(2 a)+1=2 f(a)^{2}$ so $f(2 a)-1 \neq 0$. Choose a complex $\alpha$ such that $2 \alpha^{2}(f(2 a)-1)$ $=1$ and let $g(x)=f(x+a)-f(x-a)$ and $m(x)=f(x)+\alpha g(x)$ for $x \in G$. We need only show that (2) and (3) hold. Since $f$ is even, $g$ is odd, i.e. $g(-x)=-g(x)$, $x \in G$. Hence (2) clearly holds.

To check (3), first note that, for any $x, y \in G$,

$$
\begin{aligned}
2 f(x) g(y)+ & 2 f(y) g(x)=2 f(x) f(y+a)-2 f(x) f(y-a) \\
& +2 f(y) f(x+a)-2 f(y) f(x-a) \\
= & f(x+y+a)+f(x-y-a)-f(x+y-a)-f(x-y+a) \\
& +f(y+x+a)+f(y-x-a)-f(y+x-a)-f(y-x+a) \\
= & 2 f(x+y+a)-2 f(x+y-a)=2 g(x+y)
\end{aligned}
$$

where we have used (1), the fact that $f$ is even and the definition of $g$. Thus

$$
g(x+y)=f(x) g(y)+f(y) g(x) \text { for } x, y \in G .
$$

For every $x, y \in G$,

$$
\begin{aligned}
2 g(x) g(y)= & 2[f(x+a)-f(x-a)][f(y+a)-f(y-a)] \\
= & 2 f(x+a) f(y+a)-2 f(x+a) f(y-a) \\
& -2 f(x-a) f(y+a)+2 f(x-a) f(y-a) \\
= & f(x+y+2 a)+f(x-y)-f(x+y)-f(x-y+2 a) \\
& -f(x+y)-f(x-y-2 a)+f(x+y-2 a)+f(x-y) \\
= & 2 f(x-y)-2 f(x+y)+2 f(x+y) f(2 a)-2 f(x-y) f(2 a) \\
= & 2(f(2 a)-1)(f(x+y)-f(x-y)) .
\end{aligned}
$$

That is,

$$
g(x) g(y)=(f(2 a)-1)(f(x+y)-f(x-y)) .
$$

For all $x, y \in G, f(x) f(y)=\{f(x+y)+f(x-y)\} / 2$, so from (4) and (5) we find

$$
\begin{aligned}
m(x) m(y)= & {[f(x)+\alpha g(x)][f(y)+\alpha g(y)] } \\
= & f(x) f(y)+\alpha^{2} g(x) g(y)+\alpha[f(x) g(y)+f(y) g(x)] \\
= & \left\{(1 / 2)+\alpha^{2}(f(2 a)-1)\right\} f(x+y) \\
& +\left\{(1 / 2)-\alpha^{2}(f(2 a)-1)\right\} f(x-y)+\alpha g(x+y) \\
= & f(x+y)+\alpha g(x+y)=m(x+y)
\end{aligned}
$$

by our choice of $a$ and $\alpha$. Thus (3) holds and the proof is complete.

Kannappan [3] actually proved that if $G$ is a (not necessarily abelian) group and $f$ is a complex-valued function defined on $G$ such that $f(x y z)=f(x z y)$ and $f(x y)+f\left(x y^{-1}\right)=2 f(x) f(y)$ for all $x, y, z \in G$ then there exists a complex-valued function $m$ defined on $G$ such that $f(x)=\left\{m(x)+m\left(x^{-1}\right)\right\} / 2$ and $m(x y)=$ $m(x) m(y)$ for all $x, y \in G$.

However, if $f(x y z)=f(x z y)$ for all $x, y, z \in G$ then $f(y z)=f(z y)$ for all $y, z \in G$ and therefore

$$
f((x y) z)=f(z(x y))=f(z(y x))
$$


and

$$
f(x(y z))=f((y z) x)=f(y(z x))=f(y x z) .
$$

That is $f(x y z)=f(u v w)$ whenever $x, y, z \in G$ and $(u, v, w)$ is a permutation of $(x, y, z)$. If $f(x y)+f\left(x y^{-1}\right)=2 f(x) f(y)$ for all $x, y \in G$ then, as before, $f \equiv 0$ or $f(e)=1$ ( $e$ being the identity of $G$ ) and $f\left(y^{-1}\right)=f(y)$ for all $y \in G$.

Thus the proof of Theorem 2 can be used to prove Kannappan's theorem. In fact, with only a change in notation, the proof of Theorem 2 can be used to prove

THEOREM 3. Let $G$ be a group and let $x \mapsto x^{*}$ be a mapping of $G$ to itself such that $\left(x^{*}\right)^{*}=x$ and $(x y)^{*}=x^{*} y^{*}\left(\right.$ or $\left.y^{*} x^{*}\right)$ for all $x, y \in G$. Let $f$ be a complex-valued function defined on $G$ such that $f(x y z)=f(x z y)$ for all $x, y, z \in G$. Then

$$
f(x y)+f\left(x y^{*}\right)=2 f(x) f(y) \text { for all } x, y \in G
$$

if and only if there exists a complex-valued function $m$ defined on $G$ such that $f(x)=\left\{m(x)+m\left(x^{*}\right)\right\} / 2$ and $m(x y)=m(x) m(y)$ for all $x, y \in G$.

\section{Stability.}

Theorem 4. Let $\delta>0$, let $G$ be an abelian group and let $f$ be a complex-valued function defined on $G$ such that

$$
|f(x+y)+f(x-y)-2 f(x) f(y)| \leqslant \delta \text { for all } x, y \in G .
$$

Then either $|f(x)| \leqslant(1+\sqrt{1+2 \delta}) / 2$ for all $x \in G$ or there exists a complex-valued function $m$ on $G$ such that

$$
f(x)=\{m(x)+m(-x)\} / 2 \text { for all } x \in G
$$

and

$$
|m(x+y)-m(x) m(y)| \leqslant \delta / 2 \text { for all } x, y \in G .
$$

The proof will use the following lemmas.

Lемма 1. With the assumptions of Theorem 4, $|f(0)|<\varepsilon$ where $\varepsilon=(1+\sqrt{1+2 \delta}) / 2$.

Proof. Put $x=y=0$ in (6) to get, with $z=f(0)$, that $\left.|| z|-| z\right|^{2}|<| z-z^{2} \mid<$ $\delta / 2$. It follows that $|z| \leqslant \varepsilon$.

LEMMA 2. With the assumptions of Theorem 4, $|f(2 x)|>2|f(x)|^{2}-\mu$ for all $x \in G$ where $\mu=\delta+\varepsilon$.

Proof. For any $x \in G$, so

$$
\left|f(2 x)+f(0)-2 f(x)^{2}\right|<\delta
$$

$$
|f(2 x)| \geqslant\left|2 f(x)^{2}-f(0)\right|-\delta>2|f(x)|^{2}-|f(0)|-\delta>2|f(x)|^{2}-\varepsilon-\delta .
$$

LEMMA 3. With the assumptions of Theorem 4, if $|f(x)|>\varepsilon$ for some $x \in G$ then $\left|f\left(2^{n} x\right)\right| \rightarrow+\infty$ as $n \rightarrow+\infty$.

Proof. Let $x \in G$ and $y=|f(x)|=\varepsilon+p$ for some $p>0$. Then

$$
\begin{aligned}
2 y^{2}-y-\mu & =2(\varepsilon+p)^{2}-\varepsilon-p-\delta-\varepsilon=2\left(\varepsilon^{2}-\varepsilon\right)+(4 \varepsilon-1) p+2 p^{2} \\
& =\delta+(4 \varepsilon-1) p+2 p^{2}>3 p
\end{aligned}
$$


since $\varepsilon>1$ and $2\left(\varepsilon^{2}-\varepsilon\right)=\delta$. Hence $2 y^{2}-\mu>y+3 p$. Thus, by Lemma 2 ,

$$
|f(2 x)|>2|f(x)|^{2}-\mu>|f(x)|+3 p>\varepsilon+2 p .
$$

It follows by induction that $\left|f\left(2^{n} x\right)\right|>\varepsilon+2^{n} p$ for $n=1,2,3, \ldots$

Proof of Theorem 4. Assume that $|f(x)|>\varepsilon$ for some $x \in G$. By Lemma $3, f$ is unbounded.

Put $y=0$ in (6) to conclude that $2|f(x)| \cdot|1-f(0)|<\delta$ for all $x \in G$. Since $f$ is unbounded it follows that $f(0)=1$.

For any $x, y \in G$,

$$
\begin{aligned}
2|f(x)| \cdot|f(y)-f(-y)|= & |2 f(x) f(y)-2 f(x) f(-y)| \\
< & |2 f(x) f(y)-f(x+y)-f(x-y)| \\
& +|f(x+y)+f(x-y)-f(x) f(-y)| \\
\leqslant & 2 \delta
\end{aligned}
$$

as we see by replacing $y$ by $-y$ in (6). Since $f$ is unbounded it follows that $f(-y)=f(y)$ for all $y \in G$.

Since $f$ is unbounded and $\left|f(2 x)+f(0)-f(x)^{2}\right|<\delta$ for all $x \in G$, we may choose $a \in G$ and a complex $\alpha$ such that $2 \alpha^{2}(f(2 a)-1)=1$. Let $g(x)=f(x+a)$ $-f(x-a)$ and $m(x)=f(x)+\alpha g(x)$ for $x \in G$. Since $f$ is even, $g$ is odd and therefore (2) holds.

Let $2 f(x) f(y)=f(x+y)+f(x-y)+E(x, y)$ so that $|E(x, y)|<\delta$ for all $x, y$ $\in G$. As in the proof of Theorem 2,

so

$$
\begin{aligned}
2 f(x) g(y)+2 f(y) g(x)= & 2 g(x+y)+E(x, y+a)-E(x, y-a) \\
& +E(y, x+a)-E(y, x-a)
\end{aligned}
$$

$$
|g(x+y)-f(x) g(y)-f(y) g(x)|<2 \delta \text { for } x, y \in G \text {. }
$$

Similarly

$$
|g(x) g(y)-(f(a)-1)(f(x+y)-f(x-y))|<3 \delta
$$

and

so that

$$
|f(x) f(y)-\{f(x+y)+f(x-y)\} / 2|<\delta / 2
$$

$$
\begin{aligned}
\mid m(x+y)- & m(x) m(y) \mid \\
= & \mid f(x+y)+\alpha g(x+y)-f(x) f(y) \\
\quad & -\alpha^{2} g(x) g(y)-\alpha f(x) g(y)-\alpha f(y) g(x) \mid \\
< & |\alpha(g(x+y)-f(x) g(y)-f(y) g(x))| \\
& \quad+\left|f(x+y)-f(x) f(y)-\alpha^{2} g(x) g(y)\right| \\
< & 3|\alpha| \delta+\left|f(x+y)-\{f(x+y)+f(x-y)\} / 2-\alpha^{2} g(x) g(y)\right|+\delta / 2 \\
< & 3|\alpha| \delta+\delta / 2+\mid\{f(x+y)-f(x-y)\} / 2 \\
& \quad-\left.\alpha^{2}(f(a)-1)(f(x+y)-f(x-y))|+2| \alpha\right|^{2} \delta \\
= & \delta\left(3|\alpha|+|\alpha|^{2}+1 / 2\right) \quad \text { for all } x, y \in G
\end{aligned}
$$

by choice of $a$ and $\alpha$. 
Notice that $a$ can be chosen so that $|f(a)|$ (and hence $|f(2 a)|$ ) is as large as desired. Thus $\alpha$ can be chosen so that $|\alpha|$ is as small as we wish. Thus we must have

$$
|m(x+y)-m(x) m(y)|<\delta / 2 \text { for all } x, y \in G .
$$

This completes the proof.

From Theorems 1, 2 and 4 we easily obtain

THEOREM 5. Let $\delta>0$, let $G$ be an abelian group and let $f$ be a complex-valued function on $G$ such that

$$
|f(x+y)+f(x-y)-2 f(x) f(y)|<\delta
$$

for all $x, y \in G$. Then either $|f(x)|<(1+\sqrt{1+2 \delta}) / 2$ for all $x \in G$ or

$$
f(x+y)+f(x-y)=2 f(x) f(y) \text { for all } x, y \in G .
$$

If we let $f(x)=(1+\sqrt{1+2 \delta}) / 2$ for all $x \in G$ then (6) holds but (1) fails. Thus the estimate in Theorem 5 is optimal. Also notice that if $f$ is sufficiently uniformly small or sufficiently uniformly close to 1 then (6) holds.

Using well-known results concerning the cosine equation (see e.g. [3]) we obtain the following corollary to Theorem 5 .

COROLlary. Let $\delta>0$ and let $f$ be a complex-valued, Lebesgue measurable function defined on $R^{n}$ such that

$$
|f(x+y)+f(x-y)-2 f(x) f(y)|<\delta
$$

for all $x, y \in R^{n}$. Then either $|f(x)|<(1+\sqrt{1+2 \delta}) / 2$ for all $x \in R^{n}$ or $f(x)=$ $\cos (c \cdot x)$ for all $x \in R^{n}$ where $c$ is an n-tuple of complex numbers and $c \cdot x=$ $\sum_{i-1}^{n} c_{i} x_{i}$ if $c=\left(c_{1}, \ldots, c_{n}\right)$ and $x=\left(x_{1}, \ldots, x_{n}\right)$.

\section{REFERENCES}

1. John Baker, J. Lawrence and F. Zorzitto, The stability of the equation $f(x+y)=f(x) f(y)$, Proc. Amer. Math. Soc. 74 (1979), 242-246.

2. D. H. Hyers, On the stability of the linear functional equation, Proc. Nat. Acad. Sci. U.S.A. 27 (1941), 222-224.

3. Pl. Kannappan, The functional equation $f(x y)+f\left(x y^{-1}\right)=2 f(x) f(y)$ for groups, Proc. Amer. Math. Soc. 19 (1968), 69-74.

4. S. M. Ulam, A collection of mathematical problems, Interscience, New York, 1960.

5. W. H. Wilson, On certain related functional equations, Bull. Amer. Math. Soc. 26 (1919), 300-312. Department of Pure Mathematics, Universtty of Waterloo, Waterloo, Ontario, Canada N2L 3G1 